\title{
Current status of and future strategies for multimodality treatment of unresectable stage III nonsmall cell lung cancer
}

\author{
Rudolf M. Huber ${ }^{1,4}$, Martin Reck ${ }^{2,4}$ and Michael Thomas 3

\section{Affiliations:} \\ 'Division of Respiratory Medicine and Thoracic Oncology, Dept of Medicine, University of Munich - Campus \\ Innenstadt, and Thoracic Oncology Centre Munich, Munich, \\ ${ }^{2}$ Dept of Thoracic Oncology, Hospital Grosshansdorf, Grosshansdorf, and \\ ${ }^{3}$ Internistische Onkologie der Thoraxtumoren, Thoraxklinik im Universitätsklinikum Heidelberg, Heidelberg, \\ Germany. \\ ${ }^{4}$ German Centre of Lung Research.
}

\section{Correspondence:}

R.M. Huber, Sektion Pneumologie und Thorakale Onkologie, Klinikum der LMU München, Ziemssenstraße 1 , 80336 München, Germany.

E-mail: huberlamed.uni-muenchen.de

ABSTRACT Stage III nonsmall cell lung cancer (NSCLC) encompasses a heterogeneous group of patients, some of whom may be candidates for potentially curative surgery, although for the majority surgery is not an option. Recommended therapy for patients with unresectable stage III disease is concurrent treatment with chemotherapy and thoracic radiotherapy, although even with this dual modality therapy survival remains disappointing. Novel classes of agents including targeted therapies have been shown to improve survival in advanced stage NSCLC, raising the possibility that these agents may have benefits in multimodal therapy when combined with chemoradiotherapy. Here we consider the rationale for combining new agents with chemoradiotherapy and the evidence from clinical studies assessing multimodal strategies for the management of patients with unresectable stage III NSCLC.

@ERSpublications

The rationale for combining new agents with chemoradiotherapy to treat stage III NSCLC and data from clinical studies http://ow.ly/nuE1d 


\section{Introduction}

Nonsmall cell lung cancer (NSCLC) accounts for the majority $(\sim 85 \%)$ of cases of lung cancer, which is responsible for an estimated 1.4 million deaths each year [1]. When NSCLC is diagnosed at an early stage, when the tumour is still localised (stage I/II), surgery offers a potential cure. However, local spread or metastasis is often already present at the time of diagnosis, partly accounting for the overall poor prognosis. Patients with distant metastasis or pleural effusion (stage IV) have 2-year survival rates $<10 \%$ [2] and treatment is palliative using platinum-based chemotherapy doublets, potentially in combination with targeted agents $[3,4]$.

Stage III disease occupies an intermediate position between resectable stage I-II disease and unresectable stage IV disease, and encompasses a heterogeneous group of patients that vary in terms of prognosis, tumour stage and treatment. Surgical resection is an option for selected patients. Clinical practice on resectability for stage IIIA disease tends to follow national guidelines, but those without mediastinal involvement (TNM (tumour, node, metastasis) staging T3N1M0) are generally considered resectable, while possible resectability in the presence of mediastinal involvement (T1-3N2M0) is the topic of considerable debate, with randomised controlled trials generally showing no significant difference in overall survival compared with radiotherapy/chemoradiotherapy [5-8]. Stage IIIB disease is generally considered unresectable due to the involvement of contralateral nodes, although selected patients with T4N2M0 tumours and single position N2 involvement may be candidates for resection. The combination of chemotherapy and/or radiotherapy with surgery as part of multimodal management is an area of active research and considerable debate [9-11] but is beyond the scope of the current review.

The current standard of care for patients with unresectable stage III NSCLC is concurrent treatment with platinum-based chemotherapy and thoracic radiotherapy $[4,12,13]$. Randomised clinical trials and metaanalyses have generally shown trends in favour of combination chemoradiotherapy compared with radiotherapy alone [14-23], as well as concomitant compared with sequential chemoradiotherapy [24-30]. However, many patients are considered unsuitable for chemoradiotherapy, particularly with concurrent administration, due to poor performance status or the presence of serious comorbidities.

While the data available provide general support for concurrent chemoradiotherapy, many important questions remain. The combination of agents, the total dose delivered and the schedule of administration must all be considered in order to optimise the chemotherapy component, but there are currently insufficient data in all these areas. Both induction and consolidation strategies have been investigated, but neither has demonstrated consistent benefits $[26,31,32]$ and attempts to establish an effective maintenance therapy in this setting have so far proved unsuccessful.

There is also a paucity of data regarding the optimal strategy for radiotherapy. Studies have administered different radiation doses according to different schedules [33], including hyperfractionated accelerated radiotherapy [18, 34, 35], but without providing sufficient data to reach firm conclusions. Continuous hyperfractionated accelerated radiotherapy (CHART/CHARTWEL) has failed to show a consistent survival benefit compared with conventional fractionated radiotherapy, when combined with chemotherapy in clinical trials [18, 35, 36] and increases the risk of acute toxicity, particularly oesophagitis [37]. Newer techniques, such as stereotactic, intensity-modulated and image-guided radiotherapy, have not been adequately assessed as a component of multimodal therapy for advanced disease and, as such, conventional fractionated radiotherapy remains the standard treatment. The potential benefit of prophylactic cranial irradiation to prevent brain metastases following chemoradiotherapy for NSCLC also requires further investigation [38].

Recently, advances in our understanding of the molecular pathology of NSCLC have identified new targets and strategies that may complement established therapies. A key challenge is to investigate how these possible new treatments can be best used alongside current modalities to improve outcomes for patients. This narrative review examines the available evidence on a multimodal therapeutic approach with emerging and established treatments.

\section{New strategies in multimodality therapy}

There is a pressing need to improve the management of patients with unresectable stage III NSCLC. With current standard treatment, local tumour control is suboptimal, distant metastases are common and longterm survival remains poor [30]. At present, it is not possible to predict which patients will benefit from chemoradiation, although some trials indicate that a response to induction chemotherapy is required in order to benefit from further chemotherapy during radiotherapy [39].

A range of new therapeutic options is currently under investigation as part of a multimodality approach to management of NSCLC, including both novel radiotherapy techniques and new biological agents. 
This raises further questions regarding how emerging treatment options should be used alongside chemoradiotherapy in a rational manner.

\section{New agents: current evidence}

A number of new classes of agents have been or are currently being investigated as potential components of multimodality therapy for unresectable stage III NSCLC. With the introduction of new classes of agents that act in different ways and against different targets, the ability to identify those patients most likely to benefit from a particular targeted therapy will be increasingly important. Various oncogenic mutations have been identified as potential molecular biomarkers that can predict the response to targeted therapies, including epidermal growth factor receptor $(E G F R)$ mutation status or $\mathrm{H}$-score [40], anaplastic lymphoma kinase $(A L K)$ translocation [41, 42] and c-Met amplification [43], and may play an important role in guiding treatment choices for personalised multimodal therapy [44].

\section{Inhibitors of the EGFR pathway}

There is a good theoretical rationale for combining EGFR inhibition with radiotherapy. Upregulation of signalling through the EGFR pathway is implicated in various pro-oncogenic processes, including cell proliferation, invasion, survival and angiogenesis. EGFR is frequently overexpressed in patients with NSCLC ( $\sim 30-80 \%$ of patients) and ionising radiation activates EGFR signalling by cancer cells, potentially reducing the sensitivity to radiotherapy [45]. Therefore, EGFR inhibitors might have synergistic effects when combined with radiotherapy, sensitising tumours to radiotherapy in addition to direct antiproliferative effects. In terms of scheduling, this argues for EGFR inhibition concurrent with radiotherapy, although preclinical evidence suggests that maintenance EGFR inhibition after radiotherapy may be beneficial in controlling residual disease associated with relatively radioresistant clones [46].

Somatic EGFR mutations are found in $10-20 \%$ of Caucasian patients with lung cancer and predominantly involve mutations that cause constitutive activation of the EGFR tyrosine kinase. Tumours with these mutations are highly sensitive to EGFR tyrosine kinase inhibitors (TKIs), which have been shown to be more effective than standard chemotherapy for patients with EGFR-mutated NSCLC [47]. Furthermore, NSCLC tumours with tyrosine kinase domain mutations show enhanced sensitivity to radiotherapy [48]. Therefore, multimodality therapy including radiotherapy and a TKI might be particularly beneficial in patients with EGFR-sensitising mutations.

Acquired resistance to targeted therapies is a problem in NSCLC, as in other cancers, and develops in nearly all patients over time. The mechanisms underlying acquired resistance are believed to involve activation of alternative tyrosine kinase pathways, increased angiogenesis and constitutive activation of downstream mediators. Various strategies aimed at overcoming acquired resistance, including combined inhibition of EGFR with a monoclonal antibody and a TKI, have failed. Recently, however, dual targeting of EGFR with a monoclonal antibody (cetuximab) and a pan-ErbB blocker (afatinib) has emerged as a promising approach for patients with NSCLC and acquired resistance to EGFR TKIs [49].

EGFR inhibitors that have been evaluated in combination with radiotherapy or chemoradiotherapy in the treatment of NSCLC include the monoclonal antibody cetuximab and the TKIs gefitinib and erlotinib (table 1). The data are limited but provide some preliminary insights.

\section{Cetuximab}

Preclinical data support the rationale for combining radiotherapy with cetuximab, demonstrating synergistic antitumour activity against human squamous cell carcinoma xenografts derived from the head and neck, apparently involving inhibition of post-radiation damage repair and angiogenesis as well as antiproliferative effects [70]. Similarly, in xenografts of human EGFR-overexpressing NSCLC cell lines, the combination of cetuximab and radiotherapy markedly enhanced tumour growth inhibition compared with either modality alone and had similar activity to chemoradiotherapy. The greatest inhibition of tumour growth was seen with triple combination treatment with radiotherapy, cetuximab and chemotherapy, although the differences compared with radiotherapy combined with either cetuximab or chemotherapy were not significant [71].

The effects of adding cetuximab to radiotherapy for treatment of locally advanced squamous cell carcinoma of the head and neck was assessed in a randomised clinical study in which patients received radiotherapy for 6-7 weeks, either alone $(n=211)$ or with cetuximab $\left(n=213\right.$; weekly doses of cetuximab $400 \mathrm{mg} \cdot \mathrm{m}^{-2}$ concurrent with radiotherapy, followed by seven weekly doses of cetuximab $250 \mathrm{mg} \cdot \mathrm{m}^{-2}$ ). Median overall survival was significantly improved with the combined modality treatment compared with radiotherapy alone (49.0 versus 29.3 months, hazard ratio $0.73 ; \mathrm{p}=0.018$ ). The type and incidence of adverse reactions 


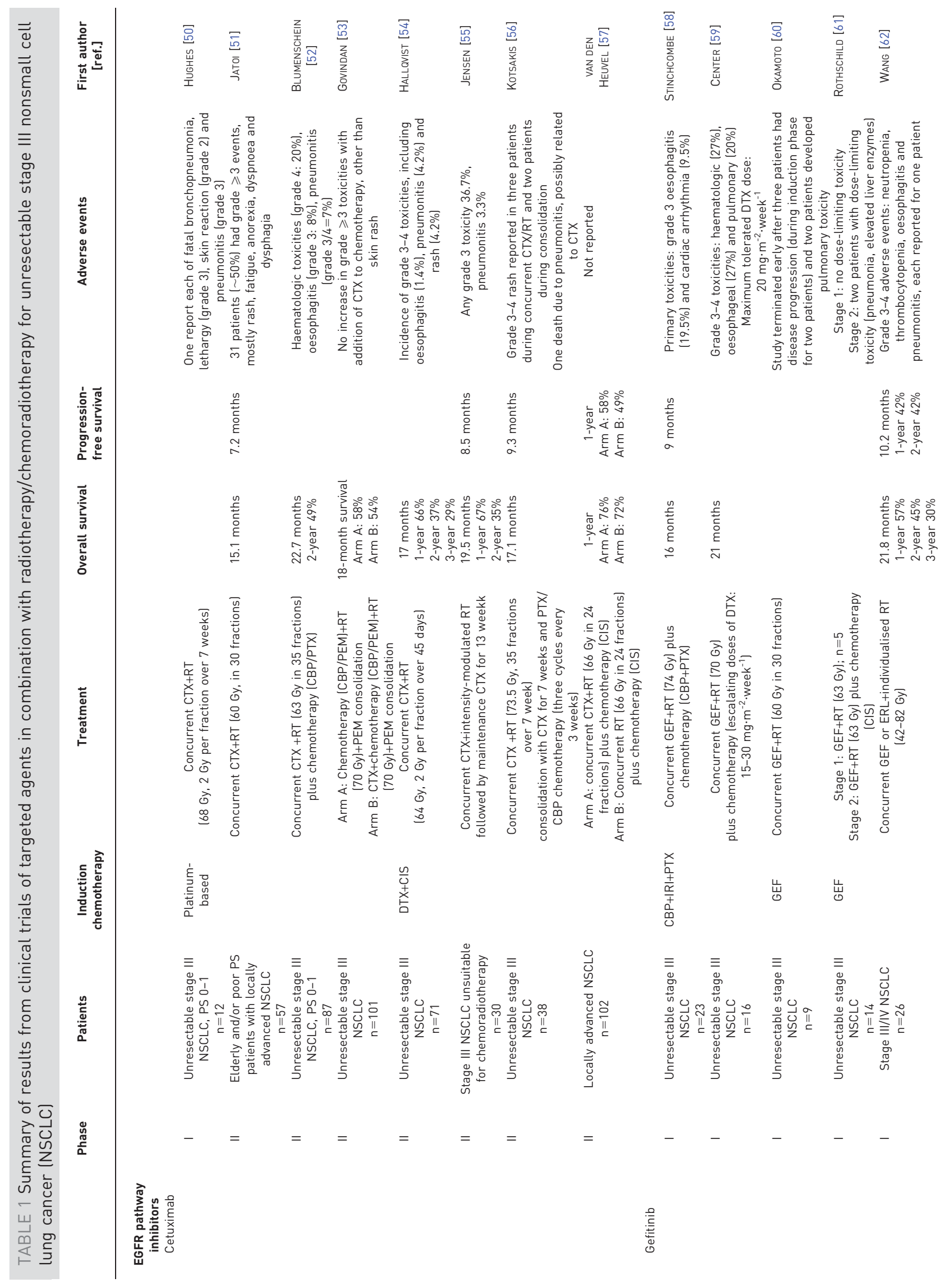




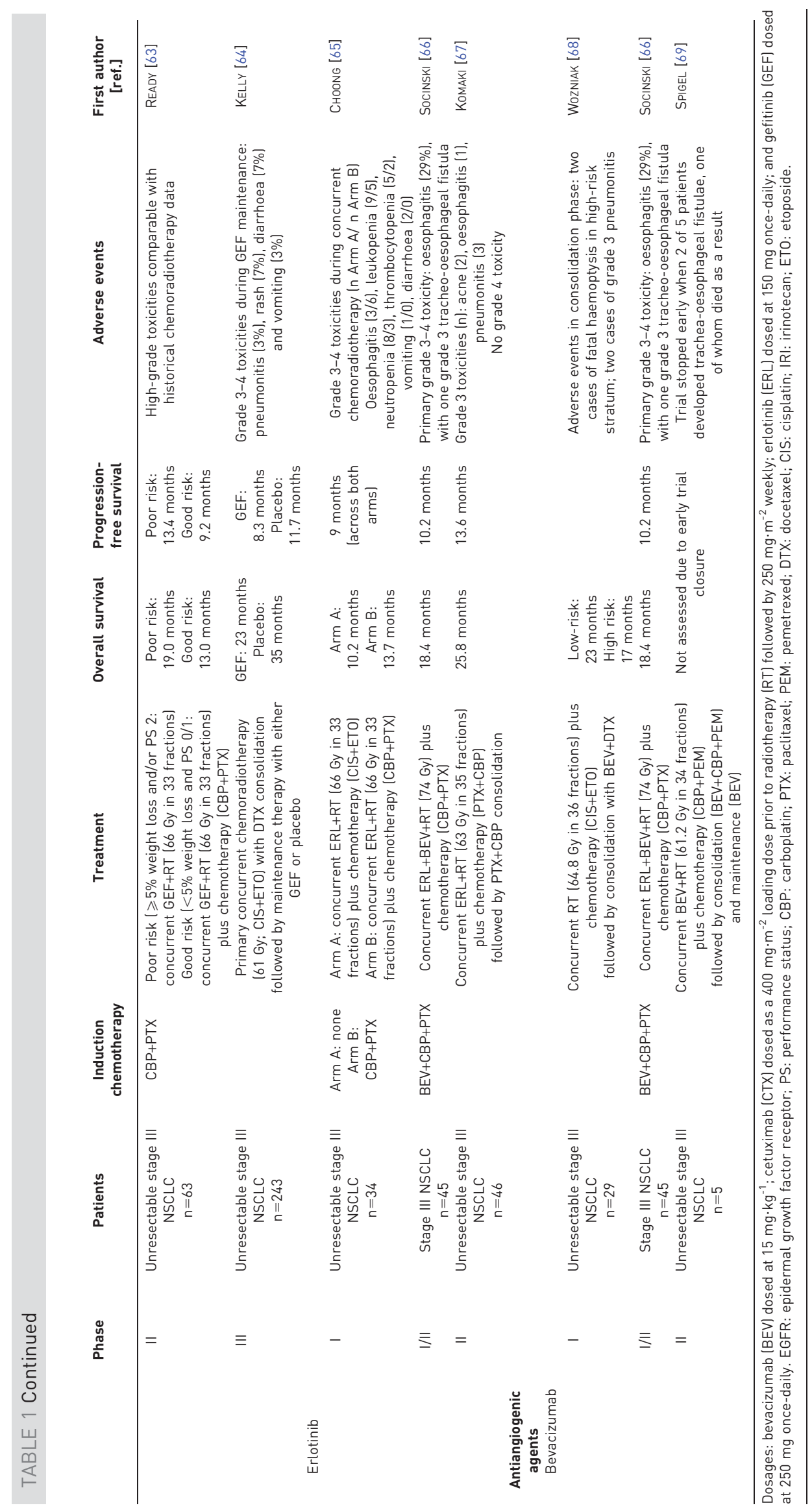


were similar, other than acneiform rash and infusion reactions which occurred more frequently when cetuximab was added to radiotherapy [72].

The results of phase I/II studies combining cetuximab with radiotherapy for stage III NSCLC suggest this is a feasible strategy, with acceptable safety and promising survival data (table 1); overall survival ranged from 15 to 23 months and toxicity appeared no worse than with chemoradiotherapy [50, 51, 54-56]. Recent studies investigating the combination of cetuximab with chemoradiotherapy suggest that this trimodal strategy is at least feasible $[52,53,57]$. In a single arm study $(n=87)$, the median survival of 22.7 months was promising [52]. A randomised study comparing chemoradiotherapy either with or without concurrent cetuximab found no additional survival benefit with cetuximab (18-month survival of 54\% and 58\%, respectively; $n=101$ ). The addition of cetuximab to chemoradiotherapy did not substantially worsen toxicity [53]. Another randomised phase II study recently reported no difference in objective control at 24 weeks with cetuximab compared with concurrent cisplatin and radiotherapy (66 Gy) alone, although long-term disease control and survival data are not yet available [57]. Ongoing studies of cetuximab in combination with radiotherapy or chemoradiotherapy are summarised in table 2.

\section{Gefitinib}

Gefitinib increases the radiosensitivity of NSCLC cell lines [73, 74], at least in part by suppressing DNA repair mechanisms [74]. In a prospective study of 26 patients with stage III/IV NSCLC treated with either gefitinib $250 \mathrm{mg}$ or erlotinib $150 \mathrm{mg}$ daily plus concurrent radiotherapy (70 Gy), median overall survival and progression-free survival (PFS) were 10.2 and 21.8 months. Safety was acceptable, with manageable acute skin, haematological, oesophageal and pulmonary toxicities [62]. Another study of concurrent gefitinib and radiotherapy for unresectable stage III NSCLC was terminated after two out of seven patients withdrew following pulmonary toxicity. EGFR-sensitising mutations were detected in two patients, both of whom had a partial response to study treatment and overall survival $>5$ years. The authors concluded that, while the combination was not worth pursuing in unselected patients, it may warrant further investigation in patients with locally advanced NSCLC and EGFR-sensitising mutations [60].

Clinical studies have also investigated the combination of gefitinib with chemoradiotherapy. Treatment of 23 patients with unresectable stage III disease with two cycles of carboplatin/irinotecan/paclitaxel induction chemotherapy followed by concurrent treatment with gefitinib (250 mg.day ${ }^{-1}$, starting on day 41), chemotherapy (carboplatin/paclitaxel) and three-dimensional conformational radiotherapy (74 Gy) resulted in median overall survival and PFS of 16 and 9 months. The primary grade 3 toxicities were oesophagitis (19.5\%) and cardiac arrhythmia (9.5\%) [58].

In another study, 16 patients with unresectable stage III NSCLC and good performance status (0-1) were treated concurrently with gefitinib $\left(250 \mathrm{mg} \cdot \mathrm{day}^{-1}\right)$ plus three-dimensional conformational radiotherapy (70 Gy) and docetaxel (escalating doses of $15-30 \mathrm{mg} \cdot \mathrm{m}^{-2}$ weekly). Patients received consolidation therapy with two cycles of docetaxel $75 \mathrm{mg} \cdot \mathrm{m}^{-2}$ and maintenance therapy with gefitinib. Median survival was 21 months and the overall response rate was $46 \%$. However, toxicity was disappointing with relatively high rates of grade 3-4 oesophageal (27\%) and pulmonary (20\%) toxicities, particularly with higher doses of docetaxel $\left(25 \mathrm{mg} \cdot \mathrm{m}^{-2}\right)$ [59]. Dose-limiting toxicities (neutropenic pneumonia and elevated liver enzymes) were also encountered in two out of nine patients in another phase I study of gefitinib administered concurrently with radiotherapy and cisplatin chemotherapy [61].

The phase II CALGB 30106 study $(n=63)$ investigated different multimodal treatment strategies according to the patient's risk level. Poor-risk patients (weight loss $\geqslant 5 \%$ and/or performance status 2 ) were treated with induction chemotherapy followed by concurrent gefitinib and radiotherapy (66 Gy). After induction chemotherapy, good-risk patients (weight loss $<5 \%$ and performance status $0-1$ ) received gefitinib concurrently with the same radiotherapy and chemotherapy (paclitaxel/carboplatin). Results for the combination of gefitinib with chemoradiotherapy were disappointing and, surprisingly, median overall survival was longer in the poor-risk compared with the good-risk patients (19 months, 95\% CI 9.9-28.4 months versus 13 months, 95\% CI 6.4-25.2 months). There were no survival differences between patients with or without EGFR-sensitising or KRAS mutations [63].

Hopes that gefitinib might be an effective option for maintenance therapy after chemoradiotherapy for stage III NSCLC have not been met. In the phase III SWOG S0023 trial, 243 patients received concurrent radiotherapy $(61 \mathrm{~Gy})$ and chemotherapy (cisplatin/etoposide) followed by three cycles of consolidation therapy with docetaxel. Patients without disease progression were then randomly assigned to maintenance therapy with gefitinib or placebo. Survival was worse with gefitinib maintenance than with placebo (median survival 23 and 35 months, respectively; $p=0.013$ ), with more rapid tumour progression, rather than gefitinib toxicity, apparently accounting for the difference in survival [64]. 


\section{Erlotinib}

Radiosensitising effects of erlotinib have been demonstrated against a range of human tumour cell lines, including NSCLC. Erlotinib appears to act at multiple levels to enhance radiosensitivity, including induction of cell cycle arrest/senescence and apoptosis, as well as inhibition of cellular repopulation and DNA damage repair $[75,76]$.

A phase I study evaluated erlotinib alongside two chemoradiotherapy regimens in patients with unresectable stage III NSCLC: 1) erlotinib plus radiotherapy (66 Gy) plus cisplatin/etoposide chemotherapy followed by 3 cycles of docetaxel consolidation; and 2) carboplatin/paclitaxel induction therapy followed by erlotinib plus radiotherapy (66 Gy) plus carboplatin/paclitaxel. The addition of erlotinib to chemoradiotherapy did not appear to increase toxicity but survival was disappointing with both regimens (median survival of 10.2 and 13.7 months) [65].

More recent results from a study in which all 46 patients received erlotinib concurrently with radiotherapy (63 Gy) and chemotherapy (carboplatin/paclitaxel) are more promising. Median overall survival was 25.8 months, with 1-year and 2-year survival rates of $84 \%$ and $75 \%$, while PFS was 13.6 months. Toxicity was acceptable, with three reports of grade 3 pneumonitis, two reports of grade 3 acne and one report of grade 3 oesophagitis [67].

Also reported recently were the results of a phase I-II trial which evaluated combining the TKI activity of erlotinib with the antiangiogenic effects of bevacizumab alongside chemoradiotherapy. Patients $(n=45)$ with unresectable stage III NSCLC received induction therapy with carboplatin/paclitaxel and bevacizumab followed by concurrent radiotherapy (74 Gy) and chemotherapy (carboplatin/paclitaxel with bevacizumab and erlotinib). Consolidation therapy with erlotinib and bevacizumab was also planned but proved unfeasible. Median overall survival and PFS were 19 months and 10 months, with objective response rates to induction and overall treatment of 39\% and 60\%, respectively. Grade 3-4 oesophagitis was reported in $29 \%$ of the patients and one patient developed a grade 3 tracheo-oesophageal fistula. The investigators concluded that the multimodal regimen appeared to offer no survival benefit at the cost of a substantial risk of, for example, oesophagitis and so is not recommended [66].

\section{Antiangiogenic agents}

Ionising radiation induces the expression of a range of pro-angiogenic factors, including vascular endothelial growth factor (VEGF), and it appears that radiation-induced upregulation of signalling via the VEGF receptor (VEGFR) pathway may contribute to radiotherapy failure by enhancing the rate of vascular repair [77]. Sensitisation of tumour cells to radiotherapy has been demonstrated with both monoclonal antibodies directed against VEGFR [78] and VEGFR TKIs [79, 80].

A number of antiangiogenic agents have been or are currently being investigated as potential therapies in the management of NSCLC, including both monoclonal antibodies (e.g. bevacizumab) and multi-targeted TKIs (e.g. sunitinib, sorafenib and vandetanib).

\section{Bevacizumab}

Bevacizumab has been evaluated in combination with chemoradiotherapy for stage III NSCLC, with disappointing results. A trial of bevacizumab combined with radiotherapy and chemotherapy (pemetrexed/ carboplatin), followed by consolidation with bevacizumab and pemetrexed/carboplatin, and then bevacizumab maintenance was stopped early when two of the five patients with unresectable NSCLC developed trachea-oesophageal fistulae. High rates of trachea-oesophageal fistulae were seen in a similar trial in patients with small-cell lung cancer [69] and high rates of ulceration and bleeding have been seen when combining bevacizumab with chemoradiotherapy in other tumour types [81, 82].

The risk of fistula formation appears to be particularly related to the combination of bevacizumab with radiotherapy, as similar rates have not been seen in studies in which bevacizumab was administered with chemotherapy only [83]. It seems likely that the risk relates to inhibition of healing of mucosal injury in the radiation field owing to the antiangiogenic effects of bevacuzimab.

Use of bevacizumab as consolidation after, rather than concurrently with, radiotherapy might theoretically reduce toxicity and the risk of fistulation. However, this strategy proved unsuccessful in a recently reported pilot study in which chemoradiotherapy for unresectable stage III NSCLC was followed by consolidation with bevacizumab and docletaxel. Patients were stratified into low- and high-risk strata based on squamous histology, haemoptysis or the presence of tumours with cavitation or near a major blood vessel. Median overall survival was 23 and 17 months for the low- and high-risk patients, respectively. Both strata were closed early, the high-risk group following two cases of fatal haemoptysis and the low-risk group due to slow accrual [68]. 


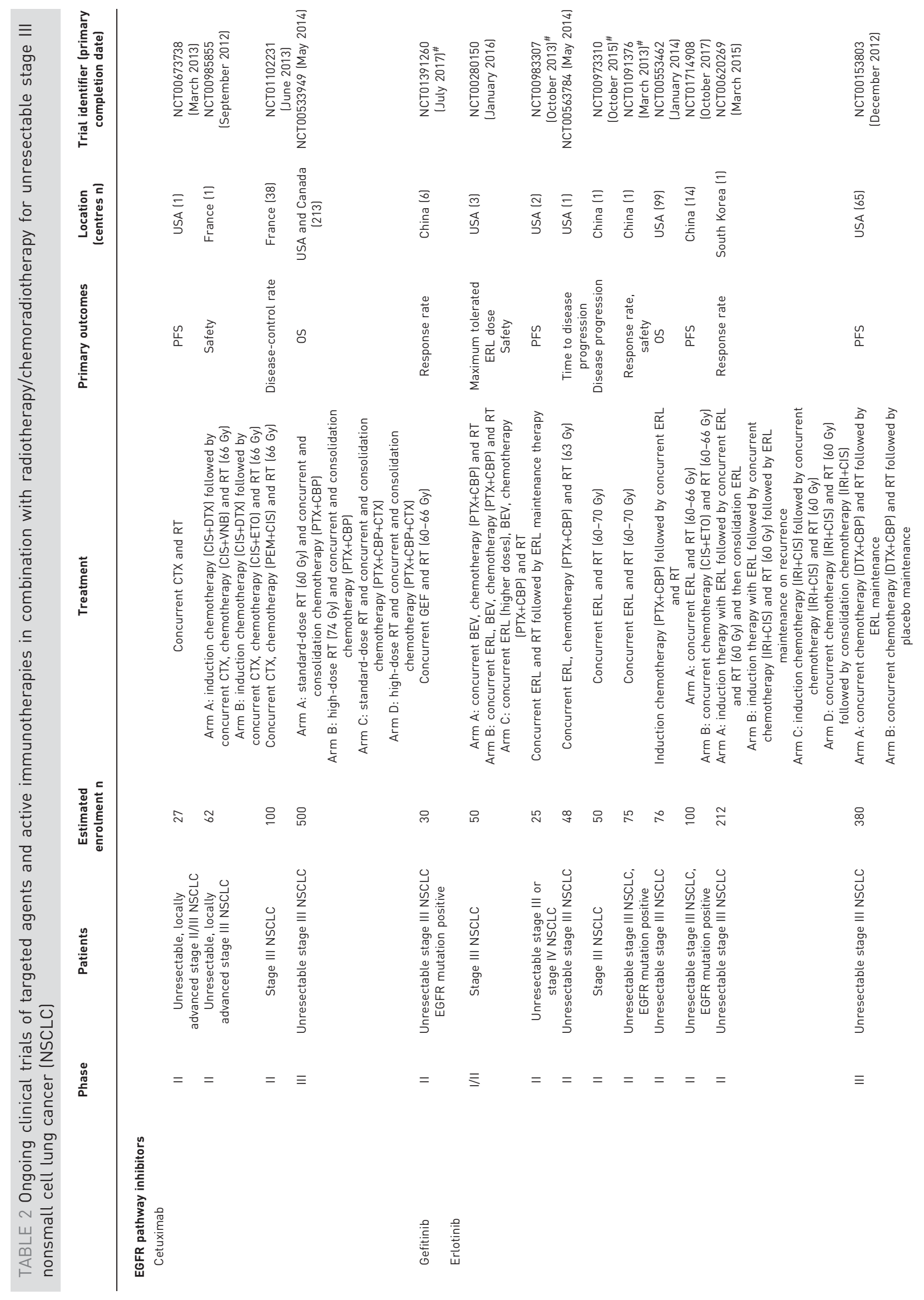




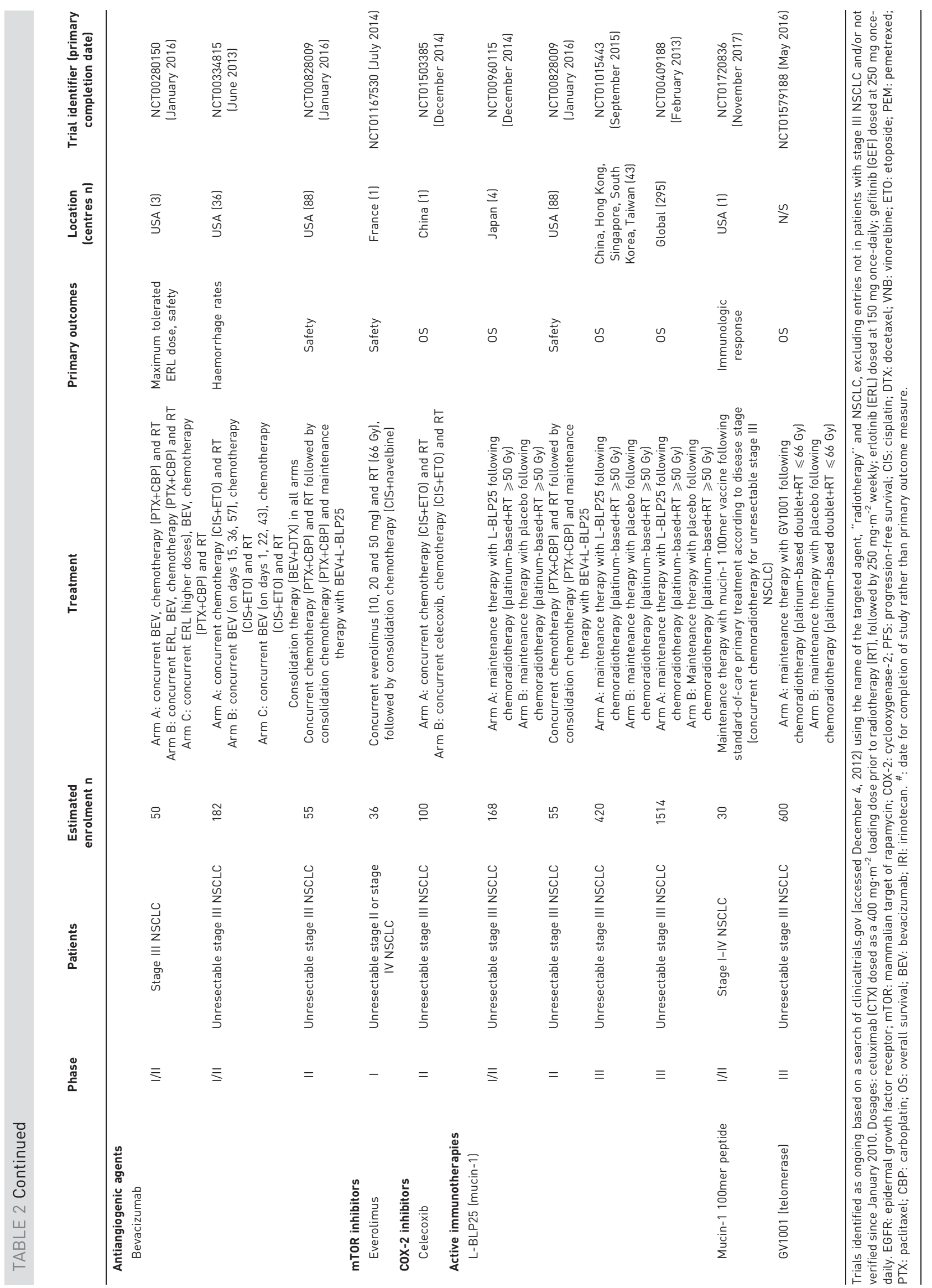


Consequently, it appears that bevacizumab is not suitable for use alongside radiotherapy. Furthermore, the results urge caution when investigating other antiangiogenic agents in combination with radiotherapy or chemoradiotherapy, particularly when planning scheduling and dosing.

\section{Multi-targeted TKIs}

Currently, there are no clinical data on the use of small molecule VEGFR TKIs in combination with radiotherapy or chemoradiotherapy for stage III NSCLC, although preclinical findings provide some support for the strategy. Radiosensitising effects of vandetanib, a TKI that inhibits both the VEGFR and EGFR pathways, have been demonstrated in an orthotopic mouse model of NSCLC. Tumour dissemination into the chest wall was common in the orthotopically injected mice, as it is in patients with NSCLC. Combination therapy with vandetanib and radiotherapy markedly reduced tumour dissemination into the thoracic wall and was more effective than either modality alone or the combination of paclitaxel with radiotherapy [84]. Vandetanib also has radiosensitising effects on head and neck carcinoma cell lines and xenografts [85-87].

Sunitinib inhibits signalling through both the platelet-derived growth factor receptor (PDGFR) and VEGFR pathways and has been shown to increase the sensitivity of a human pancreatic carcinoma cell line to radiation damage. Adding sunitinib to radiation delayed tumour growth by 30 days, compared with just 6 days with sunitinib alone and 10 days with radiation alone [88]. Another dual PDGFR/VEGFR TKI sorafenib did not appear to affect the radiosensitivity of a human colorectal cancer cell line, but tumour growth of xenografts was substantially slower with the combination of sorafenib and radiation compared with either modality alone [89].

\section{mTOR inhibitors}

The mammalian target of rapamycin (mTOR) is a serine/threonine kinase that plays an important role in regulating various basic cellular functions, including cell growth and proliferation and DNA repair. Combining the mTOR inhibitor everolimus with radiation resulted in synergistic antitumour effects in a xenograft model of human NSCLC, reducing the mean tumour volume by $73 \%$ compared with control, a significantly greater reduction than with either everolimus alone $(38 \% ; \mathrm{p}<0.001)$ or radiation alone $(52 \%$; $\mathrm{p}=0.015$ ) [90]. Addition of the mTOR inhibitor everolimus to radiation treatment has also been shown to enhance radiation damage to colon and pancreatic cancer cell lines and improved tumour control in vivo [91]. The radiosensitising effects of mTOR inhibitors appear to operate primarily on the vascular endothelium [91, 92]. Radiation recall syndrome may be a concern when combining mTOR inhibitors with radiotherapy, based on cases in which patients received mTOR inhibitors (everolimus or temsirolimus) as part of combination therapy following radiotherapy for breast, prostate or ovarian cancer [93].

\section{Cyclo-oxygenase inhibitors}

Selective cyclo-oxygenase (COX)-2 inhibition has been shown to increase the sensitivity of lung cancer cell lines to both radiation [94-96] and various chemotherapeutic agents including docetaxel and irinocetan [97]. The combination of the COX-2 inhibitor celecoxib with radiotherapy appears to be a feasible strategy in patients with NSCLC. Safety was acceptable in a phase I study including a cohort of patients with locally advanced NSCLC who received celecoxib at doses up to $800 \mathrm{mg} \cdot \mathrm{day}^{-1}$ concurrent with palliative radiotherapy (45 Gy). The main toxicities were grade 1-2 nausea and oesophagitis [98]. In a phase I-II trial, 18 patients with unresectable stage IIA or stage III NSCLC and poor performance status (performance status

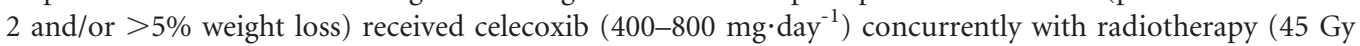
in 15 fractions or 60-66 Gy in 30-33 fractions), followed by maintenance therapy with celecoxib. Median survival was 10 months and 1- and 2-year survival rates were $44 \%$ and $22 \%$. Toxicity was comparable to that typical of radiotherapy alone [99].

A phase II trial of celecoxib $\left(800 \mathrm{mg} \cdot \mathrm{day}^{-1}\right)$ with concurrent paclitaxel/cisplatin chemoradiotherapy followed by celecoxib maintenance was stopped early due to a disappointing overall response rate. Out of 14 patients, one patient had a complete response and five patients had a partial response, resulting in an overall objective response rate of $42.9 \%$. Complete and partial responses were seen only in patients with robust reductions in the major metabolite of prostaglandin in the first 5 days after starting celecoxib treatment, suggesting that this may provide a biomarker for response to COX-2 inhibition in NSCLC. The main toxicities of the multimodal therapy were pulmonary and gastrointestinal, with one case of high-grade $(\geqslant 3)$ pneumonitis and two cases of high-grade dysphagia/oesophagitis/odynophagia [100].

\section{Active immunotherapy}

A number of active immunotherapies (therapeutic cancer vaccines) are under investigation in NSCLC. These include an allogeneic vaccine derived from irradiated NSCLC cell lines (belagenpumatucel-L), 
protein-specific vaccines (human recombinant epidermal growth factor, purified melanoma-associated antigen (MAGE)-A3 recombinant protein and telomerase) and mucin-1 targeting antigen-specific cancer immunotherapies (L-BLP25 and TG4010) [101]. Each immunotherapy differs in its mechanism of antigenic stimulus, although many incorporate immunoadjuvant to potentiate the immune response [101]. Examples include L-BLP25, administered with the adjuvant monophosphoryl lipid A, which is currently being investigated following primary chemoradiotherapy for unresectable NSCLC in the phase III START (Stimulating Targeted Antigenic Responses To NSCLC) study (table 2) [102], and MAGE-A3 with AS02B as an adjuvant, following complete resection of stage IB, II or IIIA NSCLC in the phase III MAGRIT (MAGE-A3 as Adjuvant, Non-Small Cell Lung Cancer Immunotherapy) trial [103]. As adjuvants have potent immunostimulatory effects, it is important to consider the combined effects of administering immunoadjuvant alongside other therapies in the context of multimodality regimens.

There are currently no clinical data on the concurrent use of active immunotherapy with radiotherapy. However, clinical trials in other tumour types, including prostate cancer [104] and glioblastoma [105], provide a proof of principle. Irradiation of tumours induces a cascade of pro-immunogenic effects involving both the innate and adaptive immune responses [106-108] and preliminary evidence suggests radiotherapy might enhance the effects of immunotherapy with L-BLP25 [102].

\section{Conclusions}

Rational, evidence-based decision making in the treatment of unresectable stage III NSCLC is a challenge, given that critical questions regarding optimisation of each of the individual modalities remain unanswered. Furthermore, the areas of uncertainty multiply when the different modalities are combined together and new agents are introduced into the therapeutic armamentarium.

While chemotherapy concurrent with radiation therapy is now the standard-of-care for unresectable stage III NSCLC, no single regimen can be considered as standard. Ongoing studies continue to try and determine the optimal combinations of chemotherapeutic agents, the most effective technique for delivering radiotherapy and the ideal schedules for administering radiotherapy alongside chemotherapy.

Targeted therapies have shown some initial promise in multimodality therapy, but robust evidence of consistent benefits in unresectable stage III NSCLC remain lacking. Consequently, they currently have no indication in routine practice in this setting and further investigations are needed.

Increasing attention is focused on personalising therapy, taking into account various factors including patient-related characteristics, tumour histology, treatment history and the presence of comorbidities. With the introduction of targeted therapies that act through discrete molecular pathways, the use of biomarkers to predict those patients most likely to benefit from a particular therapy is an active area of research. The best characterised and most useful of these biomarkers is currently EGFR mutation status, but other markers, such as KRAS mutations and $A L K$ translocations, may also prove valuable in guiding treatment decisions.

In order to improve the management of patients with unresectable stage III NSCLC, a number of key questions remain. In our opinion, these include the development of prognostic biomarkers, where the emphasis should be upon identifying candidates for higher intensities of systemic therapy, in the context of chemoradiotherapy regimens. In addition, a greater appreciation of the interactions between radiotherapy and systemic therapy, and the biological consequences of combining these two modalities at different intensities, is required. A primary focus of future research should include the use of near in vivo models to develop a "molecular signature" for unresectable stage III tumours. This would facilitate the treatment of tumours according to molecular status and, through the use of repeated biopsies, allow therapy to be adapted according to early treatment responses. These steps should ultimately allow personalised and flexible treatment regimens to become a reality for an increasing number of patients. Organ co-culture systems more closely model the multicell, three-dimensional tumour environment and can be used to differentiate the effects of combining radiotherapy with systemic treatments on malignant and non-malignant tissue [109]. These in vitro approaches should be included in the assessment of all new systemic agents.

With regards to priorities for clinical studies, the EGFR TKIs gefitinib and erlotinib, and the ALK inhibitor crizotinib are already used in the treatment of stage IV NSCLC patients. A first step could be to initiate phase I-II trials in which patients with appropriate genetic alterations receive the systemic agent as induction therapy and then concurrent chemoradiotherapy. The experience gained from these studies, including molecular analysis of follow-up biopsies, and clinical results, including morphological and metabolic imaging, could then be used in the design of phase III studies. Further steps in developing multimodal treatment will depend on the results of these investigations. 


\section{Acknowledgements}

Editorial assistance, including drafting and revision of the manuscript as directed by the authors, was provided by I. Faulkner (International Medical Press, Oxford, UK) funded by Merck KGaA (Darmstadt, Germany).

\section{References}

1 Ferlay J, Shin HR, Bray F, et al. Estimates of worldwide burden of cancer in 2008: GLOBOCAN 2008. Int J Cancer 2010; 127: 2893-2917.

2 Schiller JH, Harrington D, Belani CP, et al. Comparison of four chemotherapy regimens for advanced non-smallcell lung cancer. N Engl J Med 2002; 346: 92-98.

3 D’Addario G, Früh M, Reck M, et al. Metastatic non-small-cell lung cancer: ESMO Clinical Practice Guidelines for diagnosis, treatment and follow-up. Ann Oncol 2010; 21: Suppl. 5, v116-v119.

4 National Comprehensive Cancer Network. NCCN Clinical Practice Guidelines in Oncology: Non-small cell lung cancer. www.nccn.org/professionals/physician_gls/pdf/nscl.pdf Date last updated: April 12, 2012. Date last accessed: December 5, 2012.

5 van Meerbeeck JP, Kramer GW, Van Schil PE, et al. Randomized controlled trial of resection versus radiotherapy after induction chemotherapy in stage IIIA-N2 non-small-cell lung cancer. J Natl Cancer Inst 2007; 99: 442-450.

6 Johnstone DW, Byhardt RW, Ettinger D, et al. Phase III study comparing chemotherapy and radiotherapy with preoperative chemotherapy and surgical resection in patients with non-small-cell lung cancer with spread to mediastinal lymph nodes (N2); final report of RTOG 89-01. Radiation Therapy Oncology Group. Int J Radiat Oncol Biol Phys 2002; 54: 365-369.

7 Bakir M, Fraser S, Routledge T, et al. Is surgery indicated in patients with stage IIIa lung cancer and mediastinal nodal involvement? Interact Cardiovasc Thorac Surg 2011; 13: 303-310.

8 Albain KS, Swann RS, Rusch VW, et al. Radiotherapy plus chemotherapy with or without surgical resection for stage III non-small-cell lung cancer: a phase III randomised controlled trial. Lancet 2009; 374: 379-386.

9 De Craene S, Surmont V, van Meerbeeck JP. Adjuvant or neoadjuvant chemotherapy in minimal N2 stage IIIA nonsmall cell lung cancer. Curr Opin Oncol 2010; 22: 102-111.

10 Song WA, Zhou NK, Wang W, et al. Survival benefit of neoadjuvant chemotherapy in non-small cell lung cancer: an updated meta-analysis of 13 randomized control trials. J Thorac Oncol 2010; 5: 510-516.

11 Carbone DP, Felip E. Adjuvant therapy in non-small cell lung cancer: future treatment prospects and paradigms. Clin Lung Cancer 2011; 12: 261-271.

12 Jett JR, Schild SE, Keith RL, et al. Treatment of non-small cell lung cancer, stage IIIB: ACCP evidence-based clinical practice guidelines (2nd edition). Chest 2007; 132: Suppl. 3, 266S-276S.

13 Crinò L, Weder W, van Meerbeeck J, et al. Early stage and locally advanced (non-metastatic) non-small-cell lung cancer: ESMO Clinical Practice Guidelines for diagnosis, treatment and follow-up. Ann Oncol 2010; 21: Suppl. 5, v103-v115.

14 Schaake-Koning C, van den Bogaert W, Dalesio O, et al. Effects of concomitant cisplatin and radiotherapy on inoperable non-small-cell lung cancer. N Engl J Med 1992; 326: 524-530.

15 Blanke C, Ansari R, Mantravadi R, et al. Phase III trial of thoracic irradiation with or without cisplatin for locally advanced unresectable non-small-cell lung cancer: a Hoosier Oncology Group protocol. J Clin Oncol 1995; 13: $1425-1429$.

16 Trovó MG, Minatel E, Franchin G, et al. Radiotherapy versus radiotherapy enhanced by cisplatin in stage III nonsmall cell lung cancer. Int J Radiat Oncol Biol Phys 1992; 24: 11-15.

17 Jeremic B, Shibamoto Y, Acimovic L, et al. Randomized trial of hyperfractionated radiation therapy with or without concurrent chemotherapy for stage III non-small-cell lung cancer. J Clin Oncol 1995; 13: 452-458.

18 Ball D, Bishop J, Smith J, et al. A randomised phase III study of accelerated or standard fraction radiotherapy with or without concurrent carboplatin in inoperable non-small cell lung cancer: final report of an Australian multicentre trial. Radiother Oncol 1999; 52: 129-136.

19 Jeremic B, Shibamoto Y, Acimovic L, et al. Hyperfractionated radiation therapy with or without concurrent lowdose daily carboplatin/etoposide for stage III non-small-cell lung cancer: a randomized study. J Clin Oncol 1996; 14: 1065-1070.

20 Clamon G, Herndon J, Cooper R, et al. Radiosensitization with carboplatin for patients with unresectable stage III non-small-cell lung cancer: a phase III trial of the Cancer and Leukemia Group B and the Eastern Cooperative Oncology Group. J Clin Oncol 1999; 17: 4-11.

21 Bonner JA, McGinnis WL, Stella PJ, et al. The possible advantage of hyperfractionated thoracic radiotherapy in the treatment of locally advanced nonsmall cell lung carcinoma: results of a North Central Cancer Treatment Group Phase III Study. Cancer 1998; 82: 1037-1048.

22 Groen HJ, van der Leest AH, Fokkema E, et al. Continuously infused carboplatin used as radiosensitizer in locally unresectable non-small-cell lung cancer: a multicenter phase III study. Ann Oncol 2004; 15: 427-432.

23 Aupérin A, Le Péchoux C, Pignon JP, et al. Concomitant radio-chemotherapy based on platin compounds in patients with locally advanced non-small cell lung cancer (NSCLC): a meta-analysis of individual data from 1764 patients. Ann Oncol 2006; 17: 473-483.

24 Clamon G, Herndon J, Eaton W, et al. A feasibility study of extended chemotherapy for locally advanced non-small cell lung cancer: a phase II trial of cancer and leukemia group B. Cancer Invest 1994; 12: 273-282.

25 Furuse K, Fukuoka M, Kawahara M, et al. Phase III study of concurrent versus sequential thoracic radiotherapy in combination with mitomycin, vindesine, and cisplatin in unresectable stage III non-small-cell lung cancer. J Clin Oncol 1999; 17: 2692-2699.

26 Belani CP, Choy H, Bonomi P, et al. Combined chemoradiotherapy regimens of paclitaxel and carboplatin for locally advanced non-small-cell lung cancer: a randomized phase II locally advanced multi-modality protocol. J Clin Oncol 2005; 23: 5883-5891.

27 Ulutin HC, Güden M, Oysul K, et al. Split-course radiotherapy with or without concurrent or sequential chemotherapy in non-small cell lung cancer. Radiat Med 2000; 18: 93-96. 
28 Fournel P, Robinet G, Thomas P, et al. Randomized phase III trial of sequential chemoradiotherapy compared with concurrent chemoradiotherapy in locally advanced non-small-cell lung cancer: Groupe Lyon-Saint-Etienne d'Oncologie Thoracique-Groupe Francais de Pneumo-Cancerologie NPC 95-01 Study. J Clin Oncol 2005; 23: 5910-5917.

29 Belderbos J, Uitterhoeve L, van Zandwijk N, et al. Randomised trial of sequential versus concurrent chemoradiotherapy in patients with inoperable non-small cell lung cancer (EORTC 08972-22973). Eur J Cancer 2007; 43: $114-121$.

30 Aupérin A, Le Péchoux C, Rolland E, et al. Meta-analysis of concomitant versus sequential radiochemotherapy in locally advanced non-small-cell lung cancer. J Clin Oncol 2010; 28: 2181-2190.

31 Vokes EE, Herndon JE, Kelley MJ, et al. Induction chemotherapy followed by chemoradiotherapy compared with chemoradiotherapy alone for regionally advanced unresectable stage III non-small-cell lung cancer: Cancer and Leukemia Group B. J Clin Oncol 2007; 25: 1698-1704.

32 Hanna N, Neubauer M, Yiannoutsos C, et al. Phase III study of cisplatin, etoposide, and concurrent chest radiation with or without consolidation docetaxel in patients with inoperable stage III non-small-cell lung cancer: the Hoosier Oncology Group and U.S. Oncology. J Clin Oncol 2008; 26: 5755-5760.

33 Bradley JD, Paulus R, Komaki R, et al. A randomized phase III comparison of standard-dose (60 Gy) versus highdose (74 Gy) conformal chemoradiotherapy +/- cetuximab for stage IIIA/IIIB non-small cell lung cancer: preliminary findings on radiation dose in RTOG 0617. Conference abstract: ASTRO Annual Meeting; October 2-6, 2011; Miami Beach, FL, USA. http://www.astro.org/Meetings-and-Events/2011-Annual-Meeting/Media-PressResources/BradleyLBAbstract.aspx

34 Movsas B, Scott C, Langer C, et al. Randomized trial of amifostine in locally advanced non-small-cell lung cancer patients receiving chemotherapy and hyperfractionated radiation: radiation therapy oncology group trial 98-01. J Clin Oncol 2005; 23: 2145-2154.

35 Belani CP, Wang W, Johnson DH, et al. Phase III study of the Eastern Cooperative Oncology Group (ECOG 2597): induction chemotherapy followed by either standard thoracic radiotherapy or hyperfractionated accelerated radiotherapy for patients with unresectable stage IIIA and B non-small-cell lung cancer. J Clin Oncol 2005; 23: $3760-3767$.

36 Schild SE, Stella PJ, Geyer SM, et al. Phase III trial comparing chemotherapy plus once-daily or twice-daily radiotherapy in Stage III non-small-cell lung cancer. Int J Radiat Oncol Biol Phys 2002; 54: 370-378.

37 Werner-Wasik M, Paulus R, Curran WJ Jr, et al. Acute esophagitis and late lung toxicity in concurrent chemoradiotherapy trials in patients with locally advanced non-small-cell lung cancer: analysis of the radiation therapy oncology group (RTOG) database. Clin Lung Cancer 2011; 12: 245-251.

38 Gore EM, Bae K, Wong SJ, et al. Phase III comparison of prophylactic cranial irradiation versus observation in patients with locally advanced non-small-cell lung cancer: primary analysis of radiation therapy oncology group study RTOG 0214. J Clin Oncol 2011; 29: 272-278.

39 Huber RM, Flentje M, Schmidt M, et al. Simultaneous chemoradiotherapy compared with radiotherapy alone after induction chemotherapy in inoperable stage IIIA or IIIB non-small-cell lung cancer: study CTRT99/97 by the Bronchial Carcinoma Therapy Group. J Clin Oncol 2006; 24: 4397-4404.

40 Fukuoka M, Wu YL, Thongprasert S, et al. Biomarker analyses and final overall survival results from a phase III, randomized, open-label, first-line study of gefitinib versus carboplatin/paclitaxel in clinically selected patients with advanced non-small-cell lung cancer in Asia (IPASS). J Clin Oncol 2011; 29: 2866-2874.

41 McDermott U, Iafrate AJ, Gray NS, et al. Genomic alterations of anaplastic lymphoma kinase may sensitize tumors to anaplastic lymphoma kinase inhibitors. Cancer Res 2008; 68: 3389-3395.

42 Kwak EL, Bang YJ, Camidge DR, et al. Anaplastic lymphoma kinase inhibition in non-small-cell lung cancer. N Engl J Med 2010; 363: 1693-1703.

43 Toschi L, Cappuzzo F. Clinical implications of MET gene copy number in lung cancer. Future Oncol 2010; 6: 239-247.

44 Gaughan EM, Costa DB. Genotype-driven therapies for non-small cell lung cancer: focus on EGFR, KRAS and ALK gene abnormalities. Ther Adv Med Oncol 2011; 3: 113-125.

45 Dittmann K, Mayer C, Fehrenbacher B, et al. Radiation-induced epidermal growth factor receptor nuclear import is linked to activation of DNA-dependent protein kinase. J Biol Chem 2005; 280: 31182-31189.

46 Pueyo G, Mesia R, Figueras A, et al. Cetuximab may inhibit tumor growth and angiogenesis induced by ionizing radiation: a preclinical rationale for maintenance treatment after radiotherapy. Oncologist 2010; 15: 976-986.

47 Petrelli F, Borgonovo K, Cabiddu M, et al. Efficacy of EGFR tyrosine kinase inhibitors in patients with EGFRmutated non-small-cell lung cancer: a meta-analysis of 13 randomized trials. Clin Lung Cancer 2012; 13: 107-114.

48 Das AK, Sato M, Story MD, et al. Non-small-cell lung cancers with kinase domain mutations in the epidermal growth factor receptor are sensitive to ionizing radiation. Cancer Res 2006; 66: 9601-9608.

49 Janjigian YY, Groen HJ, Horn L, et al. Activity and tolerability of afatinib (BIBW 2992) and cetuximab in NSCLC patients with acquired resistance to erlotinib and gefitinib. J Clin Oncol 2011; 29: Suppl., Abstr 7525.

50 Hughes S, Liong J, Miah A, et al. A brief report on the safety study of induction chemotherapy followed by synchronous radiotherapy and cetuximab in stage III non-small cell lung cancer (NSCLC): SCRATCH study. J Thorac Oncol 2008; 3: 648-651.

51 Jatoi A, Schild SE, Foster N, et al. A phase II study of cetuximab and radiation in elderly and/or poor performance status patients with locally advanced non-small-cell lung cancer (N0422). Ann Oncol 2010; 21: 2040-2044.

52 Blumenschein GR Jr, Paulus R, Curran WJ, et al. Phase II study of cetuximab in combination with chemoradiation in patients with stage IIIA/B non-small-cell lung cancer: RTOG 0324. J Clin Oncol 2011; 29: 2312-2318.

53 Govindan R, Bogart J, Stinchcombe T, et al. Randomized phase II study of pemetrexed, carboplatin, and thoracic radiation with or without cetuximab in patients with locally advanced unresectable non-small-cell lung cancer: Cancer and Leukemia Group B trial 30407. J Clin Oncol 2011; 29: 3120-3125.

54 Hallqvist A, Wagenius G, Rylander $\mathrm{H}$, et al. Concurrent cetuximab and radiotherapy after docetaxel-cisplatin induction chemotherapy in stage III NSCLC: satellite - a phase II study from the Swedish Lung Cancer Study Group. Lung Cancer 2011; 71: 166-172.

55 Jensen AD, Münter MW, Bischoff HG, et al. Combined treatment of nonsmall cell lung cancer NSCLC stage III with intensity-modulated RT radiotherapy and cetuximab: the NEAR trial. Cancer 2011; 117: 2986-2994. 
56 Kotsakis AP, Ramalingam SS, Tarhini AA, et al. Multicenter phase II study of cetuximab (C) with concomitant radiotherapy (RT) followed by consolidation chemotherapy (CT) in locally advanced non-small cell lung cancer (NSCLC). J Clin Oncol 2011; 29: Suppl., Abstr 7019.

57 van den Heuvel MM, Vincent $\mathrm{AD}$, Uyterlinde W, et al. A randomized, multicenter phase II study investigating additional weekly cetuximab to concurrent chemoradiotherapy in locally advanced non-small cell lung carcinoma: reporting on the efficacy. J Clin Oncol 2012; 30: Suppl., Abstr 7019.

58 Stinchcombe TE, Morris DE, Lee CB, et al. Induction chemotherapy with carboplatin, irinotecan, and paclitaxel followed by high dose three-dimension conformal thoracic radiotherapy (74 Gy) with concurrent carboplatin, paclitaxel, and gefitinib in unresectable stage IIIA and stage IIIB non-small cell lung cancer. J Thorac Oncol 2008; 3: 250-257.

59 Center B, Petty WJ, Ayala D, et al. A phase I study of gefitinib with concurrent dose-escalated weekly docetaxel and conformal three-dimensional thoracic radiation followed by consolidative docetaxel and maintenance gefitinib for patients with stage III non-small cell lung cancer. J Thorac Oncol 2010; 5: 69-74.

60 Okamoto I, Takahashi T, Okamoto H, et al. Single-agent gefitinib with concurrent radiotherapy for locally advanced non-small cell lung cancer harboring mutations of the epidermal growth factor receptor. Lung Cancer 2011; 72: 199-204.

61 Rothschild S, Bucher SE, Bernier J, et al. Gefitinib in combination with irradiation with or without cisplatin in patients with inoperable stage III non-small cell lung cancer: a phase I trial. Int J Radiat Oncol Biol Phys 2011; 80: $126-132$.

62 Wang J, Xia TY, Wang YJ, et al. Prospective study of epidermal growth factor receptor tyrosine kinase inhibitors concurrent with individualized radiotherapy for patients with locally advanced or metastatic non-small-cell lung cancer. Int J Radiat Oncol Biol Phys 2011; 81: e59-e65.

63 Ready N, Jänne PA, Bogart J, et al. Chemoradiotherapy and gefitinib in stage III non-small cell lung cancer with epidermal growth factor receptor and KRAS mutation analysis: cancer and leukemia group B (CALEB) 30106, a CALGB-stratified phase II trial. J Thorac Oncol 2010; 5: 1382-1390.

64 Kelly K, Chansky K, Gaspar LE, et al. Phase III trial of maintenance gefitinib or placebo after concurrent chemoradiotherapy and docetaxel consolidation in inoperable stage III non-small-cell lung cancer: SWOG S0023. J Clin Oncol 2008; 26: 2450-2456.

65 Choong NW, Mauer AM, Haraf DJ, et al. Phase I trial of erlotinib-based multimodality therapy for inoperable stage III non-small cell lung cancer. J Thorac Oncol 2008; 3: 1003-1011.

66 Socinski MA, Stinchcombe TE, Moore DT, et al. Incorporating bevacizumab and erlotinib in the combined-modality treatment of stage III non-small-cell lung cancer: results of a phase I/II trial. J Clin Oncol 2012; 30: 3953-3959.

67 Komaki R, Blumenschein GR, Wistuba II, et al. Phase II trial or erlitinib and radiotherapy following chemoradiotherapy for patients with stage III non-small cell lung cancer. J Clin Oncol 2011; 29: Suppl., Abstr 7020.

68 Wozniak AJ, Moon J, Thomas CR, et al. SWOG S0533: a pilot trial of cisplatin (C)/eoposide (E)/radiotherapy (RT) followed by consolidation docetaxel (D) and bevacizumab (B) (NSC-704865) in three cohorts of patients (pts) with inoperable locally advanced stage III non-small cell lung cancer (NSCLC). J Clin Oncol 2012; 30: Suppl., Abstr 7018.

69 Spigel DR, Hainsworth JD, Yardley DA, et al. Tracheoesophageal fistula formation in patients with lung cancer treated with chemoradiation and bevacizumab. J Clin Oncol 2010; 28: 43-48.

70 Huang SM, Harari PM. Modulation of radiation response after epidermal growth factor receptor blockade in squamous cell carcinomas: inhibition of damage repair, cell cycle kinetics, and tumor angiogenesis. Clin Cancer Res 2000; 6: 2166-2174.

71 Raben D, Helfrich B, Chan DC, et al. The effects of cetuximab alone and in combination with radiation and/or chemotherapy in lung cancer. Clin Cancer Res 2005; 11: 795-805.

72 Bonner JA, Harari PM, Giralt J, et al. Radiotherapy plus cetuximab for squamous-cell carcinoma of the head and neck. N Engl J Med 2006; 354: 567-578.

73 Burdak-Rothkamm S, Rübe CE, Nguyen TP, et al. Radiosensitivity of tumor cell lines after pretreatment with the EGFR tyrosine kinase inhibitor ZD1839 (Iressa). Strahlenther Onkol 2005; 181: 197-204.

74 Tanaka T, Munshi A, Brooks C, et al. Gefitinib radiosensitizes non-small cell lung cancer cells by suppressing cellular DNA repair capacity. Clin Cancer Res 2008; 14: 1266-1273.

75 Chinnaiyan P, Huang S, Vallabhaneni G, et al. Mechanisms of enhanced radiation response following epidermal growth factor receptor signaling inhibition by erlotinib (Tarceva). Cancer Res 2005; 65: 3328-3335.

76 Wang M, Morsbach F, Sander D, et al. EGF receptor inhibition radiosensitizes NSCLC cells by inducing senescence in cells sustaining DNA double-strand breaks. Cancer Res 2011; 71: 6261-6269.

77 Solberg TD, Nearman J, Mullins J, et al. Correlation between tumor growth delay and expression of cancer and host VEGF, VEGFR2, and osteopontin in response to radiotherapy. Int J Radiat Oncol Biol Phys 2008; 72: 918-926.

78 Lee CG, Heijn M, di Tomaso E, et al. Anti-vascular endothelial growth factor treatment augments tumor radiation response under normoxic or hypoxic conditions. Cancer Res 2000; 60: 5565-5570.

79 Abdollahi A, Lipson KE, Han X, et al. SU5416 and SU6668 attenuate the angiogenic effects of radiation-induced tumor cell growth factor production and amplify the direct anti-endothelial action of radiation in vitro. Cancer Res 2003; 63: 3755-3763.

80 Bischof M, Abdollahi A, Gong P, et al. Triple combination of irradiation, chemotherapy (pemetrexed), and VEGFR inhibition (SU5416) in human endothelial and tumor cells. Int J Radiat Oncol Biol Phys 2004; 60: 1220-1232.

81 Crane $\mathrm{CH}$, Eng C, Feig BW, et al. Phase II trial of neoadjuvant bevacizumab, capecitabine, and radiotherapy for locally advanced rectal cancer. Int J Radiat Oncol Biol Phys 2010; 76: 824-830.

82 Crane $\mathrm{CH}$, Ellis LM, Abbruzzese JL, et al. Phase I trial evaluating the safety of bevacizumab with concurrent radiotherapy and capecitabine in locally advanced pancreatic cancer. J Clin Oncol 2006; 24: 1145-1151.

83 Crinò L, Dansin E, Garrido P, et al. Safety and efficacy of first-line bevacizumab-based therapy in advanced nonsquamous non-small-cell lung cancer (SAiL, MO19390): a phase 4 study. Lancet Oncol 2010; 11: 733-740.

84 Shibuya K, Komaki R, Shintani T, et al. Targeted therapy against VEGFR and EGFR with ZD6474 enhances the therapeutic efficacy of irradiation in an orthotopic model of human non-small-cell lung cancer. Int J Radiat Oncol Biol Phys 2007; 69: 1534-1543.

85 Sano D, Matsumoto F, Valdecanas DR, et al. Vandetanib restores head and neck squamous cell carcinoma cells' sensitivity to cisplatin and radiation in vivo and in vitro. Clin Cancer Res 2011; 17: 1815-1827. 
86 Gustafson DL, Frederick B, Merz AL, et al. Dose scheduling of the dual VEGFR and EGFR tyrosine kinase inhibitor vandetanib (ZD6474, Zactima) in combination with radiotherapy in EGFR-positive and EGFR-null human head and neck tumor xenografts. Cancer Chemother Pharmacol 2008; 61: 179-188.

87 Yang S, Wu J, Zuo Y, et al. ZD6474, a small molecule tyrosine kinase inhibitor, potentiates the anti-tumor and antimetastasis effects of radiation for human nasopharyngeal carcinoma. Curr Cancer Drug Targets 2010; 10: 611-622.

88 Cuneo KC, Geng L, Fu A, et al. SU11248 (sunitinib) sensitizes pancreatic cancer to the cytotoxic effects of ionizing radiation. Int J Radiat Oncol Biol Phys 2008; 71: 873-879.

89 Suen AW, Galoforo S, Marples B, et al. Sorafenib and radiation: a promising combination in colorectal cancer. Int $J$ Radiat Oncol Biol Phys 2010; 78: 213-220.

90 Mauceri HJ, Sutton HG, Darga TE, et al. Everolimus exhibits efficacy as a radiosensitizer in a model of non-small cell lung cancer. Oncol Rep 2012; 27: 1625-1629.

91 Manegold PC, Paringer C, Kulka U, et al. Antiangiogenic therapy with mammalian target of rapamycin inhibitor RAD001 (Everolimus) increases radiosensitivity in solid cancer. Clin Cancer Res 2008; 14: 892-900.

92 Shinohara ET, Cao C, Niermann K, et al. Enhanced radiation damage of tumor vasculature by mTOR inhibitors. Oncogene 2005; 24: 5414-5422.

93 Bourgier C, Massard C, Moldovan C, et al. Total recall of radiotherapy with mTOR inhibitors: a novel and potentially frequent side-effect? Ann Oncol 2011; 22: 485-486.

94 Pyo H, Choy H, Amorino GP, et al. A selective cyclooxygenase-2 inhibitor, NS-398, enhances the effect of radiation in vitro and in vivo preferentially on the cells that express cyclooxygenase-2. Clin Cancer Res 2001; 7: 2998-3005.

95 Grimes KR, Warren GW, Fang F, et al. Cyclooxygenase-2 inhibitor, nimesulide, improves radiation treatment against non-small cell lung cancer both in vitro and in vivo. Oncol Rep 2006; 16: 771-776.

96 Kim BM, Won J, Maeng KA, et al. Nimesulide, a selective COX-2 inhibitor, acts synergistically with ionizing radiation against A549 human lung cancer cells through the activation of caspase-8 and caspase-3. Int J Oncol 2009; 34: 1467-1473.

97 Hida T, Kozaki K, Muramatsu H, et al. Cyclooxygenase-2 inhibitor induces apoptosis and enhances cytotoxicity of various anticancer agents in non-small cell lung cancer cell lines. Clin Cancer Res 2000; 6: 2006-2011.

98 Liao Z, Komaki R, Milas L, et al. A phase I clinical trial of thoracic radiotherapy and concurrent celecoxib for patients with unfavorable performance status inoperable/unresectable non-small cell lung cancer. Clin Cancer Res 2005; 11: 3342-3348.

99 Gore E, Bae K, Langer C, et al. Phase I/II trial of a COX-2 inhibitor with limited field radiation for intermediate prognosis patients who have locally advanced non-small-cell lung cancer: radiation therapy oncology group 0213 . Clin Lung Cancer 2011; 12: 125-130.

100 Mutter R, Lu B, Carbone DP, et al. A phase II study of celecoxib in combination with paclitaxel, carboplatin, and radiotherapy for patients with inoperable stage IIIA/B non-small cell lung cancer. Clin Cancer Res 2009; 15: 2158-2165.

101 Mellstedt H, Vansteenkiste J, Thatcher N. Vaccines for the treatment of non-small cell lung cancer: investigational approaches and clinical experience. Lung Cancer 2011; 73: 11-17.

102 Butts C, Maksymiuk A, Goss G, et al. Updated survival analysis in patients with stage IIIB or IV non-small-cell lung cancer receiving BLP25 liposome vaccine (L-BLP25): phase IIB randomized, multicenter, open-label trial. J Cancer Res Clin Oncol 2011; 137: 1337-1342.

103 GlaxoSmithKline. GSK1572932A Antigen-specific cancer immunotherapeutic as adjuvant therapy in patients with non-small cell lung cancer. http://clinicaltrials.gov/ct2/show/NCT00480025 Date last updated: July 11, 2013. Date last accessed: December 5, 2012.

104 Gulley JL, Arlen PM, Bastian A, et al. Combining a recombinant cancer vaccine with standard definitive radiotherapy in patients with localized prostate cancer. Clin Cancer Res 2005; 11: 3353-3362.

105 Muragaki Y, Maruyama T, Iseki H, et al. Phase I/IIa trial of autologous formalin-fixed tumor vaccine concomitant with fractionated radiotherapy for newly diagnosed glioblastoma. Clinical article. J Neurosurg 2011; 115: 248-255.

106 Formenti SC, Demaria S. Systemic effects of local radiotherapy. Lancet Oncol 2009; 10: 718-726.

107 Roses RE, Xu M, Koski GK, et al. Radiation therapy and Toll-like receptor signaling: implications for the treatment of cancer. Oncogene 2008; 27: 200-207.

108 Ferrara TA, Hodge JW, Gulley JL. Combining radiation and immunotherapy for synergistic antitumor therapy. Curr Opin Mol Ther 2009; 11: 37-42.

109 Edelmann M, Gamarra F, da Silva AK, et al. Cell cycle effects of radiation on human bronchial epithelium and lung carcinoma cells in monolayer cultures and a three-dimensional co-culture system. Radiat Res 2005; 164: 391-399. 